\title{
Forecast of Building Energy Consumption Using RBF Neural Network based on L-GEM
}

\author{
Yuncan Xue ${ }^{+}$, Enpeng Qiu and Juntao Fei \\ College of IoT Engineering, Hohai University, Changzhou 213022, China
}

\begin{abstract}
Building energy consumption accounts for a large part of the total energy consumption of the society. Its accurate prediction is very important for energy-saving. In order to forecast building energy consumption, RBF neural network (RBFNN) is used. Based on the discussion that the neural networks yield small error on training dataset does not always perform well on testing dataset, we proposed a method to use RBFNN by applying the Localized Generalization Error Model (L-GEM) in this paper. To obtain the optimum localized generalization error (L-GE), a mofified chaotic mutation PSO is presented to search the optimum L-GE of RBFNN. Experiments have been carried and the results show that the RBFNN based on the L-GEM outperforms general RBFNN in building energy consumption prediction.
\end{abstract}

Keywords: building energy consumption, RBF neural network; localized generalization error model.

\section{Introduction}

Building energy saving has been a hot research field for several years. It is a very important way to improve the building thermal environment, the national economic development, and the atmospheric environmental quality. The essence of building energy-saving is to improve energy utilization in buildings. Energy consumption prediction provides us an efficient way to draw up a schedule for promoting the energy utilization rate.

A great number of papers have been published on the topic of energy consumption prediction. In [1], genetic algorithms (GA) were proposed to predict the building energy consumption. In [2], a hybrid integrated architecture was put forward to improve the knowledge acquisition and make better decisions for reducing energy consumptions. Fahmy predicts the future energy consumption reduction by using GRC envelope optimization [3].

However, the neural networks $(\mathrm{NN})$ have been shown their better performance in the building energy saving and prediction research [4].Among these, the Radial Basis Function (RBF) neural network has the characteristics of fast training speed, global optimization and good generalization, etc. So, it is very suitable for solving building energy consumption prediction problems.

When solving the problem, usually a training error is minimized to find the set of parameters of the NN for good performance. However, a NN yields small error on training dataset does not always perform well on testing dataset. It is because that the training dataset may not provide enough information to the $\mathrm{NN}$ to learn. Over-fitting occurs when the size of training dataset is too small and such that the NN is able to memorize all the training samples instead of learning the underlying relationship between inputs and outputs.

The goal of training a $\mathrm{NN}$ is to recognize future unseen samples well and therefore minimizing the training error only always could not provide satisfactory results. Many different methods have been proposed

\footnotetext{
+ Corresponding author. Tel.: + 86-519-85192016; fax: +86-519-85106049.
}

E-mail address: ycxue@hhuc.edu.cn. 
to estimate the generalization error of a $\mathrm{NN}$ for a given pattern classification problem. Paper[5] gives a detail discussion of these methods and concludes that these methods less practical for NN.

The localized Generalization Error Model (L-GEM) framework was proposed [5] for evaluating the generalization capability of NN. It only considers the localized generalization error in the area around the training samples. The model is based on the idea that a trained NN usually cannot perform well for unseen samples with too many differences to the training samples. The L-GEM has been successfully applied in a wide range of applications.

However, to obtain the optimum L-GE, an intelligent optimization algorithm is necessary. A modified chaotic mutation PSO (CMPSO) algorithm is put forward to solve the problem.

The rest of this paper is organized as follows. The influence factors of building energy consumption are discussed in Section 2. Section 3 gives a design of RBF neural network (RBFNN). L-GEM is introduced in Section 4. In Section 5, a modified chaotic mutation PSO algorithm is presented and experiments of energy consumption forecast are carried and discussed. We conclude our work in Section 6.

\section{The Influence Factors of Building Energy Consumption}

The data of building energy consumption is the result of the comprehensive influence of a variety of factors. The influence factors of energy consumption can be summed up to four types:(1) building body parameters, (2) electrical equipment design parameters, (3) the climate parameters of the building, (4) management situation of the equipment.

For the former two types, however, these factors are basically determined after the building is put into operation. Therefore, from the point of view of machine learning to find the law of energy consumption, these two kinds of factors are not suitable for adoption and are longer discussed in this paper. The third type includes temperature, relative humidity, wind speed, wind direction, intensity of illumination, rainfall and so on. It is known from the literature that the temperature, relative humidity and sunshine intensity have a great influence on the energy consumption of the building. The fourth type mainly includes: air conditioning time, lighting time, indoor temperature standard, fresh air standard, personnel density, and so on. The opening or closing of the air conditioning and lighting equipment is related to whether have people there, so whether the day is a working day should be analyzed.

Based on the discussion, the following four variables are selected as the factors affecting the energy consumption data of office buildings: (1) the daily maximum temperature, (2) Sunshine intensity, (3) The relative humidity, (4) Whether the day is a working day.

Most weather websites do not record the intensity of sunshine, so it is impossible to get the data directly. By review the meteorological literature, we know that the total cloudiness is an important factor affecting sunshine intensity, and it is also an intuitive indicator reflecting the weather type. Therefore, this paper quantifies the weather types to reflect the intensity. The weather types and their corresponding quantized values are shown in the Table 1.

Table 1: Relation table of cloudiness and weather types

\begin{tabular}{ll}
\hline weather types & cloudiness \\
\hline sunny & 1 \\
cloudy & 0.9 \\
overcast & 0.85 \\
small rain (snow) & 0.75 \\
moderate rain (snow) & 0.7 \\
heavy rain (snow) & 0.65 \\
\hline
\end{tabular}

\section{Design of RBF Neural Network}

The RBF neural network (RBFNN) is an effective front feed neural network proposed by J. Moody and C. Darken in the late 1980s. It is a three layer forward network, which is divided into input layer, hidden 
layer and output layer. The input layer nodes only transfer input signals to the hidden layer, whose nodes are composed of radial effect function such as Gaussian function. The basis function of hidden layer is:

$$
\phi_{j}=\exp \left(-\frac{\left\|x-u_{j}\right\|^{2}}{2 v_{j}^{2}}\right), j=1,2, \cdots, m
$$

where, $x=\left[x_{1}, x_{2}, \cdots x_{n}\right]^{T}$ is the input of the network, $h_{j}$ is the output of the $j$ th neuron of the hidden layer, that is: $u_{j}=\left[u_{j 1}, \cdots, u_{j n}\right]$ is the center of the first $\mathrm{j}$ basis function, which has the same dimension with $x$; The width vector of the Gaussian function is $v=\left[v_{1}, \cdots, v_{m}\right]^{T}, v_{\mathrm{j}}$ is the first $j$ perception variable, it determines the width of the center of basis function; $m$ is the number of hidden nodes. The weights of the network are $w=\left[w_{1}, \ldots, w_{m}\right]^{T}$. The output layer $y_{m}(t)=w_{1} h_{1}+w_{2} h_{2}+\cdots w_{m} h_{m}$ is a linear combination of the hidden layer.

Based on the above discussion, for our building energy consumption problem, we can design our RBFNN. First, we find that the different weather types seriously affect the building energy consumption. From table 1, we can see that cloudiness can reflect all the weather types. Therefore, cloudiness, maximum temperature, relative humidity and whether the day is a working day are selected as input variables. Second, the output nodes are the forecast of building energy consumption. Last, we design the hidden layer. Hidden layer with too much nodes or too little nodes are both bad for network training. Here we design the node number according to empirical formula:

$$
\mathrm{m}=\sqrt{n+p}+a
$$

where, $n$ is the number of input nodes; $p$ is the output node number; $m$ is the number of hidden layer nodes; $a$ is the constant of $1 \sim 10$. Through repeated experiments, taking full account of time and accuracy, here the hidden layer node of neural network is selected as 8 .

\section{Localized Generalization Error Model for RBFNN}

In our problem, the ultimate goal is to train a NN to minimize the error of the unseen samples. However, it is not reasonable to expect a NN to minimize the error of the unseen samples which are very different from the training example. To solve this problem, L-GEM proposed by Yeung [5], which considers the error occurred in the neighborhoods of training samples rather than the whole input space is adopted.

Given a training sample $X(b)$, the neighborhood $S_{Q}^{b}$ of this training sample is defined as $S_{Q}^{b}=\{X=X(b)$ $+\Delta X\}$. For $\forall \Delta X$ that satisfy $0<\left|\Delta x_{\mathrm{i}}\right| \leq \mathrm{Q} \forall i=1, \ldots, N$, where $\Delta X=\left(\Delta x_{1}, \Delta x_{2}, \ldots, \Delta x_{\mathrm{N}}\right)$ and $N$ denotes the number of features of a training sample. The neighborhoods of the whole training dataset (SQ) is defined to be the union of all $S_{Q}^{b}$, where $b=1 \ldots l$ and $l$ is the number of training samples. This neighborhood is called Q-neighborhoods.

The mean square error (MSE) in the $Q$-neighborhoods $\left(S_{Q}\right)$ will be considered. We define $\operatorname{RSM}(Q)$ to be:

$$
R_{\theta}\left(S_{Q}\right)=\int_{S_{Q}}\left(F(X)-f_{\theta}(X)\right)^{2} p(X) d X
$$

with probability $(1-\eta)$, we have:

$$
\begin{aligned}
R_{\theta}\left(S_{Q}\right) & \leq \frac{1}{l} \sum_{b=1}^{l} \int_{S_{Q}^{(b)}}\left(F(X)-f_{\theta}(X)\right)^{2} p(X) d X \\
& \leq\left(\sqrt{R_{e m p}}+\sqrt{E_{S_{Q}}\left((\Delta y)^{2}\right)}+A\right)^{2}+\varepsilon \\
& =R_{\theta}^{*}\left(S_{Q}\right)
\end{aligned}
$$

Where:

$$
\begin{gathered}
R_{\text {emp }}=(1 / l) \sum_{b=1}^{l}(\text { err })^{2} \\
E_{S_{Q}}\left((\Delta y)^{2}\right)=\frac{1}{l} \sum_{b=1}^{l} \int_{S_{Q}^{(b)}}(\Delta y)^{2} \frac{1}{(2 Q)^{N}} d X \\
\Delta y=f_{\theta}(X)-f_{\theta}\left(X^{(b)}\right) \\
A=(\max (F(X))-\min (F(X))) \\
B=\max \left(\left(f_{\theta}(X)-F(X)\right)^{2}\right)
\end{gathered}
$$




$$
\varepsilon=B \sqrt{\frac{\ln \eta}{-2 l}}
$$

With $R_{\theta}^{*}\left(S_{Q}\right)$, the upper bound of the MSE can be calculated. For RBFNN, Eq.(6) can be rewritten as:

Where:

$$
\begin{aligned}
E_{S_{Q}}\left((\Delta y)^{2}\right) \approx \sum_{j=1}^{M} \varphi_{j} & \left(\left(\sum_{\mathrm{i}=1}^{n} \sigma_{\Delta x_{i}}^{2}\left(\sigma_{x_{i}}^{2}+\left(\mu_{x i}-\mu_{j i}\right)^{2}+0.2 \sigma_{\Delta x_{i}}^{2}\right)\right) / v_{j}^{4}\right) \\
= & \frac{1}{3} Q^{2} \sum_{j=1}^{M} \varphi_{\mathrm{j}}\left(E\left(S_{j}\right) / v_{j}^{4}+\frac{0.2 N}{9} Q^{4} \sum_{j=1}^{M} \varphi_{j} / v_{j}^{4}\right.
\end{aligned}
$$

$$
\begin{gathered}
\varphi_{j}=\left(\omega_{j}\right)^{2} \exp \left(\frac{\operatorname{Var}\left(s_{j}\right)}{2 v_{j}^{4}}-\frac{E\left(s_{j}\right)}{v_{j}^{2}}\right) \\
E\left(s_{j}\right)=\sum_{i=1}^{N}\left(\sigma_{x_{i}}^{2}+\left(\mu_{x_{i}}-\mu_{j i}\right)^{2}\right) \\
\operatorname{Var}\left(s_{j}\right)=\sum_{i=1}^{N}\left(E_{D}\left[\left(x_{i}-\mu_{x_{i}}\right)^{4}\right]-\left(\sigma_{x_{i}}^{2}\right)^{2}+4 E_{D}\left[\left(x_{i}-\mu_{x_{i}}\right)^{3}\right]\left(\mu_{x i}-\mu_{j i}\right)+4 \sigma_{x_{i}}^{2}\left(\mu_{x i}-\mu_{j i}\right)^{2}\right)
\end{gathered}
$$

where $\mu_{x_{i}}$ and $\sigma_{x_{i}}^{2}$ represent expectation and variance of the input feature. $\sigma_{\Delta x_{i}}^{2}$ represents the input perturbation of the $i$ th input feature.

\section{Experiment}

To use RBFNN based on the L-GEM to predict energy consumption, we must find out the optimum $R_{i}^{*}\left(S_{Q}\right)$ first. To train RBFNN with L-GEM, an intelligent optimization algorithm is necessary. Here, a modified chaotic mutation PSO (CMPSO) algorithm is used and its objective function is chosen as:

$$
\text { fitness }_{i}=R_{i}^{*}\left(S_{Q}\right)
$$

Particle swarm optimization is a population-based evolutionary computation technique developed by Kennedy and Eberhart in 1995. It has been widely used because PSO can define search direction and search scopes only based on the fitness function converted from the objective function and doesn't need to know the differential of objective function and other auxiliary information. However, PSO has such problem as local extremum, premature. In order to prevent premature appearance, the diversity of population should be maintained. Here we randomly choose some particles and let them move in the opposite direction so as to ensure the diversity of the population. In order to improve the search precision, we introduce the chaos search strategy into the PSO.

According to the above discussion, a modified chaotic mutation PSO (CMPSO) is presented. The algorithm can be summarized as follows:

Step1 Initialization. Generate particle population and find out the individual and global optima of the initial population using Eq.(15). Initialize the chaotic search variables.

Step 2 Update the particle velocity according to the mutation probability, if it is less than the mutation probability, updates the particle velocity according that the particle moves in the opposite direction, otherwise, update it using classical velocity modification formula. Update the position variables.

Step 3 Find out the fitness value of each particle using Eq.(15), and update the individual and global optima.

Step 4 For the best particle with global optima, use chaos search to get new global optima.

Step 5 Determine if the termination conditions are satisfied, if satisfied, jump to Step 7.

Step 6 Determine whether the maximum evolution generation is reached, if not, returns to Step 2.

Step 7 Terminate the optimization process and output results.

In order to show the good performance of our RBF based on the L-GEM, experiments are conducted. According to the above discussion, the data of building energy consumption and influence factors of office buildings are recorded from March 1, 2014 to April 30th, and 61 sets of sample data are formed [6]. From March 15th to March 28th, March 29th to April 11th, 28 groups of samples were selected as test sets and other samples were used as training sets. All the input values and output values are normalized to $[0,1]$. 
Fig. 1 is the fitting curve of the predicted value (red curve) and the actual value (blue curve). From the figure, it can be seen that the predicted value tracks the actual value well. The green curve in Fig. 1 is the relative errors between the test results of the sample and the actual data. The maximum relative error is $12.57 \%$, and the minimum relative error is $1.04 \%$, while the relative error is between $0.012 \%$ and $20.251 \%$ by RBFNN [6]. From the experiments, it can be seen that the RBF based on the L-GEM outperforms general RBF. It also shows that L-GEM is a suitable method in measuring the performance of NN in both seen and unseen data.

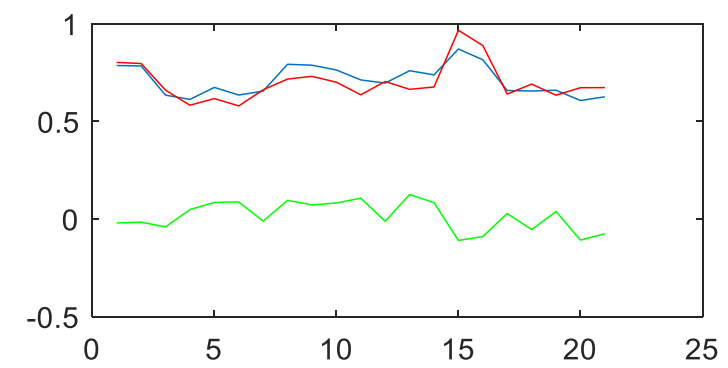

Fig. 1: Fitting curve of the predicted value and the actual value.

\section{Conclusion}

In this paper, we proposed a method to apply RBFNN based on L-GEM for building energy consumption prediction. To obtain the optimum solution of RBFNN with L-GEM, PSO algorithm is adopted. In order to prevent its premature appearance and improve its search precision, a modified chaotic mutation PSO is presented. This modified algorithm is used to search for the optimum L-GE of RBFNN. Experiments have been carried and the results show that the RBFNN using the proposed L-GEM outperforms general RBF networks in building energy consumption prediction and that L-GEM is a suitable method in measuring the performance of the neural networks in both seen and unseen data.

\section{Acknowledgements}

This work was supported by the Changzhou science and technology support plan (industry) (CE2016044).

\section{References}

[1] S. Ene, I. Kucukoglu, A. Aksoy, et al. "A genetic algorithm for minimizing energy consumption in warehouses", Energy, 114:973-980, 2016.

[2] A. Mate, J. Peral, A. Ferrandez, et al. "A hybrid integrated architecture for energy consumption prediction", Future Generation Computer Systems, 63(C):131-147, 2016.

[3] M. Fahmy, M. Mahdy, M. Nikolopoulou. "Prediction of future energy consumption reduction using GRC envelope optimization for residential buildings in Egypt", Energy \& Buildings, 70(2):186-193, 2014.

[4] R.M. Yedra, F. Rodriguez Diaz, M. Del Mar Castilla Nieto, et al. "A neural network model for energy consumption prediction of CIESOL bioclimatic building," Advances in Intelligent Systems \& Computing, 239:51-60, 2014.

[5] D.S. Yeung, W.W.Y. Ng, D. Wang, et al. "Localized generalization error model and its application to architecture selection for radial basis function neural network", IEEE Transactions on Neural Networks, 18(5):1294-1305,2007.

[6] Z P Liu, The research and design of wireless energy consumption monitoring system in building, Masteral Dissertation of Dalian University of Technology,2015. 